\title{
Epigenetic Modulation in Hematologic Malignancies: Challenges and Progress
}

\author{
Constantine S. Mitsiades, MD, PhD, and Kenneth C. Anderson, MD
}

\section{Key Words}

Epigenetics, hematologic malignancy

\begin{abstract}
Genetic alterations, including gene mutations, and chromosomal amplifications, deletions, inversions, and translocations, are hallmarks of the molecular biology of cancer. These events lead to oncogene activation, formation of chimeric oncoproteins, and/ or inactivation of tumor suppressor genes. Such genetic changes contribute to the neoplastic transformation of cells, as well as the eventual acquisition by malignant cells of a more aggressive biologic and clinical behavior. However, in recent years, it has become apparent that these genetic events are not the sole determinants of the biologic behavior of tumor cells. Indeed, it is becoming increasingly apparent that tumor cells with a given genotype exhibit a differential phenotype depending on the microenvironment in which they reside. Furthermore, extensive data have shown that derivative daughter cells of neoplastic, as well as normal cells, inherit changes in the patterns of gene expression that are not associated with changes in the primary DNA sequence but are instead related to changes in chromatin structure and its accessibility for transcriptional activity. These heritable gene expression changes that are not associated with changes in the primary nucleotide sequence are referred to as epigenetic changes. This review provides an overview of the regulation of the "epigenome" in neoplastic cells, with particular emphasis on DNA methylation and histone acetylation as therapeutic targets for hematologic malignancies. (JNCCN 2009;7[Suppl 8]:S1-S12)
\end{abstract}

Genetic alterations, including gene mutations, and chromosomal amplifications, deletions, inversions, and translocations are hallmarks of the molecular biology of cancer. ${ }^{1,2}$ These events lead to oncogene activation, formation of chimeric oncoproteins, and/or inactivation of tumor suppressor genes. Such genetic changes contribute to the neoplastic transformation of cells, as well as the eventual acquisition by malignant cells of a more aggressive biologic and clinical behavior. However, in recent years, it has become apparent that these genetic events are not the sole determinants of the biologic behavior of tumor cells. ${ }^{3}$ Indeed, it is becoming increasingly apparent that tumor cells with a given genotype exhibit a differential phenotype depending on the microenvironment in which they reside. ${ }^{4}$ Furthermore, an extensive body of data has shown that derivative daughter cells of neoplastic, as well as normal cells, inherit changes in the patterns of gene expression that are not associated with changes in the primary DNA sequence but instead are related to changes in chromatin structure and its accessibility for transcriptional activity. ${ }^{5,6}$ Such heritable gene expression changes that are not associated with changes in the primary nucleotide sequence are referred to as epigenetic changes.

This review provides an overview of the regulation of the "epigenome" in neoplastic cells, with particular emphasis on DNA methylation and histone acetylation as therapeutic targets for hematologic malignancies.

\section{Role of Epigenetics in Human Neoplasias}

The DNA inside the nucleus of both normal and malignant cells is organized into nucleosomes, which are the basic functional units of chromatin. Each nucleosome corresponds to 147 base pairs of DNA wrapped around a multimeric complex of histones $\mathrm{H} 2 \mathrm{~A}, \mathrm{H} 2 \mathrm{~B}, \mathrm{H} 3$, and $\mathrm{H} 4$ (2 copies of each histone per nucleosome). ${ }^{7} \mathrm{Nu}$ cleosomes are linked to each other by DNA sequences (approximately 160-240 base pairs in length), which interact with histone $\mathrm{H} 1$ and can be further organized to form more compact helical structures that determine the degree of packaging of the chromatin. The concept of epigenetic regulation of gene transcription was based on observations that covalent chemical posttranslational modifications of the tails of histone molecules lead to changes in gene transcription as a result of structural changes in the 3-dimensional conformation of chromatin. ${ }^{6}$ These posttranslational changes, which 
include methylation, acetylation, phosphorylation, ubiquitination, and SUMOylation, modulate the accessibility of DNA to transcription factors and other regulators of gene expression. ${ }^{6}$ In addition, these posttranslational modifications of histones interact with another important component of epigenetic modification, namely, the methylation of DNA at CpG dinucleotide sites, to determine the state of transcriptional activity of corresponding genes.

In $\mathrm{CpG}$ dinucleotide sites, where a cytosine residue precedes guanosine, the C5 position of the pyrimidine ring of cytosine can receive a methyl group, donated by S-adenosyl methionine, in a biochemical reaction catalyzed by DNA methyltransferases. $\mathrm{CpG}$ dinucleotides are not uniformly distributed in the human genome. Instead, they appear to be overrepresented in the so-called $\mathrm{CpG}$ islands, which are relatively short DNA regions $(0.5 \mathrm{~kb}$ to a few $\mathrm{kb})$ that are frequently located near promoter sequences of different genes. ${ }^{8}$ Cytosine residues in $\mathrm{CpG}$ islands, particularly those present within promoter regions, are typically not methylated in nonmalignant cells, while $\mathrm{CpG}$ dinucleotides in downstream sequences within the body of the gene and in the $3^{\prime}$ untranslated region are typically present in lower density compared with $\mathrm{CpG}$ islands, and are predominantly methylated in nonmalignant cells. The hypomethylation of the promoter region and the presence of $\mathrm{CpG}$ methylation in the body of the gene facilitate the recruitment of transcription factors, transcriptional activators, and histone acetyltransferases (HATs) in the promoter region, while methylcytosine-binding proteins and histone deacetylases (HDACs) are recruited to the methylated $\mathrm{CpG}$ dinucleotides in the body of the gene. This pattern of differential recruitment of these factors is conducive to active transcription of the corresponding gene. ${ }^{5}$ Notable exceptions to this pattern for nonmalignant cells have been described in the context of gene imprinting and in genes within the regions of X-chromosome inactivation..$^{9,10}$ In contrast to the DNA methylation pattern observed in normal cells, malignant cells exhibit a reversal in the distribution of $\mathrm{CpG}$ methylation, ${ }^{11,12}$ which involves 1) widespread hypomethylation in the body of genes, within intronic sequences, and within repetitive DNA sequences, and 2) hypermethylation in $\mathrm{CpG}$ islands within promoter regions. ${ }^{5,13-17}$

Both of these events are considered capable of contributing to the neoplastic phenotype through different, and potentially overlapping, mechanisms. Global hypomethylation has been proposed to have a negative overall effect on structural stability of chromosomal material because of increased aneuploidy (attributed to hypomethylation in centromeres), ${ }^{18,19}$ increased mitotic recombination (which predisposes to loss of heterozygosity and chromosomal rearrangements), and loss of normal imprinting patterns. ${ }^{20,21}$ In contrast, increased methylation in promoterassociated $\mathrm{CpG}$ islands is considered a key mechanism for silencing of tumor suppressor genes, such as $\mathrm{p} 21^{22,23}$ or $R b \cdot \cdot^{24,25}$ In fact, there are data to suggest that in many tumor models the frequency of hypermethylation of $\mathrm{CpG}$ islands in promoter regions of tumor suppressor genes is similar to the frequency of deletions or inactivating mutations of these genes. ${ }^{5}$ The precise mechanisms for hypermethylation of promoter region-associated $\mathrm{CpG}$ islands in tumor cells or for the apparent predilection of tumor suppressor gene promoters for this hypermethylation are not completely understood. ${ }^{3}$

Among the diverse posttranslational histone modifications that can influence gene transcription, acetylation and methylation have been studied in the greatest detail. The acetylation status of histones is regulated by the functionally opposing activities of HDACs and HATs. The latter transfer acetyl groups from acetyl-CoA to lysine residues in the histone tail, while the former facilitate the removal of these groups. The hyperacetylated state of histones has historically been thought to be associated with a structurally open chromatin and active transcription of corresponding genes, whereas histone deacetylation has been associated with suppression of gene expression and/or heterochromatin formation. ${ }^{26,27}$ This has been attributed to the fact that increased acetylation of histones neutralizes the positive charge of their lysine residues, thereby attenuating the electrostatic interaction of the nucleosome histone core with the negatively charged DNA backbone. Furthermore, it has been proposed that acetylated histones constitute sites for docking of bromodomain-containing regulatory factors necessary for transcriptional activation. ${ }^{28}$ However, the relationship between histone acetylation status and transcription of individual genes is more complex. The activation of gene transcription is determined by several different functionally opposing epigenetic events. For instance, the processes of DNA methylation and histone acetylation seemed 
Epigenetic Modulation in Hematologic Malignancies

to be functionally opposing, because DNA methyltransferases recruit HDACs to methylated $\mathrm{CpG}$ sites of gene promoters, further contributing to a transcriptionally inactive chromatin state. ${ }^{29}$ Interestingly, there are data to suggest that, in the context of this functionally opposing role of DNA methylation and histone acetylation, the former appears to play a functionally dominant role, which can keep the corresponding genes in a transcriptionally silent status, despite increased histone acetylation. ${ }^{14,30,31}$ Other posttranslational modifications of histones can influence the state of transcriptional activity of genes. For instance, methylation of histones can either activate or suppress gene transcription, depending on the particular site on the $N$-terminal histone tail, where methylation takes place. The methylation of lysine residues 9 and 27 of histone $\mathrm{H} 3$ (H3K9 and $\mathrm{H} 2 \mathrm{~K} 27$ ) is typically associated with suppression of transcription, in contrast to the methylation of lysine 4 of histone $\mathrm{H} 3$ (H3K4), which is associated with transcriptional activation. ${ }^{32,33}$ To further underscore the complexity of this regulatory system, acetylation of $\mathrm{H} 3 \mathrm{~K} 9$ facilitates the methylation of $\mathrm{H} 3 \mathrm{~K} 4$, especially in genes with unmethylated $\mathrm{CpG}$ islands. ${ }^{17,34}$ Figure 1 illustrates epigenetic inactivation of tumorsuppressor genes and agents that may be able to partially restore this distorted epigenetic picture. ${ }^{35}$

A growing number of pathways and molecular mediators critical for tumor cell proliferation, survival, drug resistance, metastatic potential, and other aspects of tumor cell biology are regulated at an epigenetic level. Many studies have been able to validate a direct relationship between increased DNA methylation and transcriptional silencing of genes known to have a negative regulatory role on cell cycle progression (e.g., Rb, p14, p15, p16, p57, p73); ${ }^{13,36-42}$ proapoptotic signaling (caspase-8, DAPK1, TMS-1);4-47 growth factor signaling (SOCS1, SOCS3, CRBP1, RARbeta2); ${ }^{39,44,48-52}$ repair of DNA damage (Fanconi anemia-BRCA pathway members, GST $\pi$, hMLH1, O6MGMT); ${ }^{13,39,53-56}$ inhibition of angiogenesis (VHL, EFEMP1, BNIP3, BNIP3L, IGFBP3, and EGLN2); ${ }^{57-59}$ or inhibition of metastatic potential (TIMP3, E-cadherin). ${ }^{44,54,60}$ In these cases, the transcriptional silencing appears to provide an advantage to the tumor cell by abrogating the expression and function of mediators that negatively affect the proliferative capacity, drug resistance, and metastatic potential of the tumor cell.
The main targets of DNA methylation in cancer cells appear to fall into 2 large categories: 1) targets of epigenetic silencing that seem to play a role in a broad spectrum of tumor neoplasias (e.g., p16, $\mathrm{Rb}),{ }^{13,36-39,41,54,61,62}$ and 2) genes silenced in a tumor type-specific manner, reflecting putative tumor suppressive roles in select tissues. ${ }^{5,14,15}$

Posttranslational modifications of histones also affect the expression and/or function of a pleiotropic spectrum of molecular pathways important for cancer cell biology. HATs and HDACs can regulate gene expression not only by affecting chromatin structure, but also by affecting the DNA binding and transcriptional activity of key transcription factors, such as p53, STAT3, ETS, and RUNX1.63,64

Furthermore, histone methylation provides an additional level of regulation of gene expression through a complex system that involves methylation of arginine (either mono- or dimethylation) or lysine (mono-, di-, or trimethylation) residues. The methylation state of histones is regulated by the opposing action of histone methyltransferases and histone demethylases. Arginine methylation is typically associated with transcriptional activation, whereas lysine methylation can be associated with transcriptional repression (when the methylation involves residues $\mathrm{H} 3 \mathrm{~K} 9, \mathrm{H} 3 \mathrm{~K} 27$, and $\mathrm{HK} 20$ ) or conversely constitutional activation (when the methylation involves residues $\mathrm{H} 3 \mathrm{~K} 4, \mathrm{H} 3 \mathrm{~K} 36$, and H3K79). ${ }^{65,66}$

\section{Epigenetic Changes as Prognostic Markers}

The significance of epigenetic changes for neoplastic cells of hematologic malignancies is underscored by 2 observations: agents targeting epigenetic regulation have shown clinical activity for diverse hematologic malignancies; aberrations in epigenetic markers have been found to correlate with clinical outcome in several clinical settings of patients with hematologic malignancies. Perhaps the most robust evidence for the prognostic significance of epigenetic changes in hematologic malignancies has come from the setting of diffuse large B-cell lymphoma, where hypomethylation of the promoter region for the DNA repair enzyme $\mathrm{O}^{6} \mathrm{MGMT}^{67}$ has been associated with significantly shorter progression-free and overall survival times among patients receiv- 


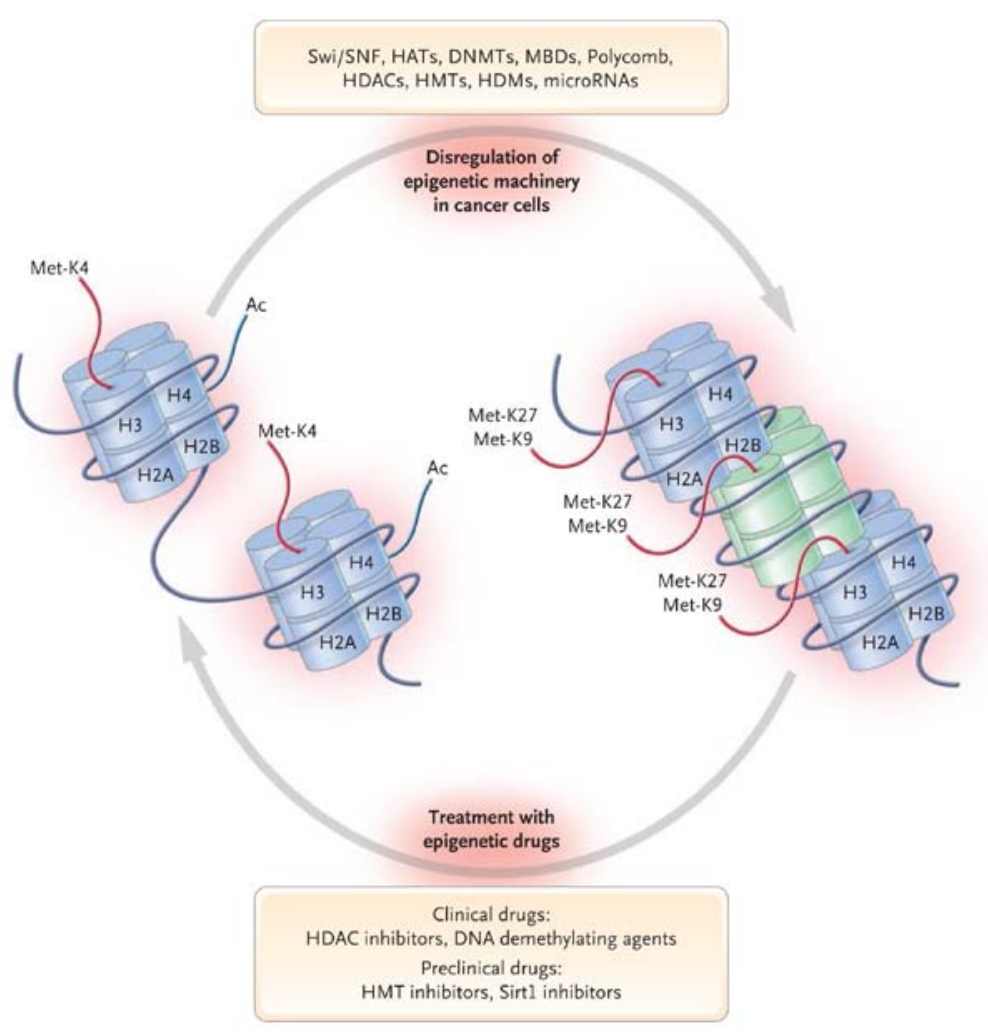

Figure 1 Epigenetic inactivation of tumor-suppressor genes. ${ }^{35}$ In a normal cell, expression of the mRNA of a tumor-suppressor gene occurs in the context of an unmethylated promoter $\mathrm{CpG}$ island and histone modification, such as hyperacetylation and methylation of lysine 4 of histone H3. Gray cylinders indicate octamers of histones, consisting of histones H2A, H2B, H3, and H4. They form the nucleosomes, and the double strand of DNA is wrapped around them. A combination of selection and targeted disruption of the DNA methylation and histone-modifier proteins disrupts the epigenetic circumstances in the cancer cell. Epigenetic inactivation of tumor-suppressor genes is associated with dense $\mathrm{CpG}$-island promoter hypermethylation and the appearance of repressive histone markers such as methylation of lysines 9 and 27 of histone H3. Epigenetic drugs can partially restore the distorted epigenetic picture by removing inactivation markers (e.g., DNA methylation) and inducing the presence of active markers (e.g., histone acetylation). Abbreviations: AC, acetylation; DNMTs, DNA methyltransferases; HATs, histone acetyltransferases; HDAC, histone deacetylase; HDMs, histone demethylases; HMTs, histone methyltransferases; MBDs, methyl-CpG-binding domain proteins; Met-K4, methylation of lysine 4; Met-K9, methylation of lysine 9; Met-K27, methylation of lysine 27; Sirt1, sirtuin 1; Swi/SNF, switching/sucrose nonfermenting chromatin-remodeling complex.

From Esteller M. Epigenetics in cancer. N Engl J Med 2008;358:1148-1159. Copyright (C) 2008. Massachusetts Medical Society. All rights reserved.

ing cyclophosphamide-based chemotherapy for this disease. This observation is compatible with similar data pertaining to response of glioma to carmustine ${ }^{68}$ and other alkylating agents (alone or with radiation therapy). ${ }^{55,56,69,70}$ In the context of leukemias, p15 methylation was shown to be an independent predictor of inferior disease-free survival in acute promyelocytic leukemia, ${ }^{71}$ while patients with acute lymphoblastic leukemia with a higher number of hypermethylated tumor suppressor genes have a worse prognosis. ${ }^{72}$ Lastly, patients with myeloma harboring hypermethylation of the promoter regions for p16 $6^{44,73,74}$ or DAPK $1^{75}$ have been reported to have an unfavorable prognosis after treatment with conventional antimyeloma therapies.

\section{Pharmacologic Modulators of Epigenetic Regulation as Therapeutics for Hematologic Neoplasias}

Currently, DNA methyltransferase inhibitors and HDAC inhibitors constitute the 2 classes of epigenetic agents that are most advanced in terms of clinical applications. Indeed, members of these 2 classes have already been approved by the FDA for various indications in hematologic malignancies. Further 
Epigenetic Modulation in Hematologic Malignancies

studies may reveal whether these classes of agents may also have activity in the context of solid tumors.

\section{DNA Methyltransferase Inhibitors}

Azacitidine (5-azacytidine) and decitabine (2'-deoxy5-azacytidine) are the 2 DNA methyltransferase inhibitors currently approved by the FDA. Other DNA methyltransferase inhibitors currently in development include zebularine, SGI-110, and RG108. The 2 FDA-approved DNA methyltransferase inhibitors, as well as several of the currently developed compounds in this class, are nucleoside analogs (one exception is the RG108 compound, which is an active site inhibitor). Azacitidine, the prototypical member of this drug class, was initially studied in the 1960s for its properties as a classical cytotoxic chemotherapeutic agent. Indeed, being an analog of cytosine, azacitidine is incorporated into the DNA and, at high doses, inhibits DNA synthesis. However, it was noted that at lower doses (1-2 logs lower than the directly cytotoxic doses of the compound) azacitidine treatment is incorporated not only into the DNA during DNA synthesis, ${ }^{76}$ but in that context, azacitidine also functions to inhibit DNA methyltransferase activity.

A phase III, international, randomized, open-label trial compared subcutaneous azacitidine treatment versus conventional care in patients with higher-risk myelodysplastic syndromes (MDS). ${ }^{77}$ Conventional care was defined as best supportive care, low-dose cytarabine, or intensive chemotherapy, as selected by investigators before randomization. Median overall survival was 24.5 months for the azacitidine group versus 15.0 months for the conventional care group (hazard ratio, 0.58; 95\% CI, 0.43-0.77; $P=.0001$ ). At last follow-up, 82 patients in the azacitidine group had died compared with 113 in the conventional care group. At 2 years, $50.8 \%$ of patients in the azacitidine group were alive compared with $26.2 \%$ in the conventional care group $(P<.0001) .{ }^{77}$ Subsequent studies of azacitidine and other DNA methyltransferase inhibitors have confirmed these observations and extended them to other clinical settings of MDS, such as studies showing that decitabine offers a survival advantage compared with intensive chemotherapy in patients with higher-risk MDS. ${ }^{78}$ Studies exploring alternative dosing and schedules of administration, ${ }^{79,80}$ as well as combination regimens with other agents, ${ }^{81-83}$ have been performed or are ongoing. Fur- thermore, the feasibility of stem cell transplant after treatment with DNA methyltransferase inhibitors has been documented. ${ }^{84}$

DNA methyltransferase inhibitors have also been tested in leukemias and myeloproliferative disorders, including combinations with imatinib in patients with chronic myeloid leukemia; ${ }^{85}$ combinations with HDAC inhibitors, such as valproic acid ${ }^{86,87}$ or phenylbutyrate, ${ }^{81}$ in acute myeloid leukemia (AML); combinations with all-trans retinoic acid; ${ }^{87}$ and combinations with hydroxyurea and gemtuzumab ozogamicin in patients with previously untreated non-M3 AML; $; 2$ as well as studies in patients with myelofibrosis ${ }^{88}$ or chronic myelomonocytic leukemia. ${ }^{89}$ The aggregate experience from these trials is that DNA methyltransferase inhibitors can be safely administered in combination with other antileukemic agents. While in the setting of MDS, DNA methyltransferase inhibitors are, as single agents, capable of improving the natural history of MDS, and have in fact become a standard of care for patients with higher-risk MDS, ${ }^{90}$ more studies are needed to evaluate whether the anti-leukemic clinical activity of combinations incorporating this drug class is superior to that of conventional regimens.

\section{HDAC Inhibitors}

The study of HDAC inhibitors in hematologic malignancies and solid tumors was preceded by early observations in the 1980s that proposed a link between the ability of sodium butyrate to induce cell differentiation in erythroleukemia cells $s^{91,92}$ and the compound's ability to trigger hyperacetylation of histones. ${ }^{93-95}$ Sodium butyrate did not receive FDA approval, but anecdotal evidence of clinical response in a butyrate-treated patient with acute myelocytic leukemia ${ }^{96}$ provided impetus for further study of other short-chain fatty acids with HDAC inhibitory activity (e.g., valproic acid). Furthermore, it provided a first level of supportive evidence for the development of new classes of HDAC inhibitors, including hydroxamates, such as vorinostat (suberoylanilide hydroxamic acid), panobinostat (LBH589), belinostat, cyclic peptides (such as FK228, also known as depsipeptide or romidepsin), or benzamides. ${ }^{97}$

The Role of HDAC Inhibitors in Cutaneous and Peripheral T-Cell Lymphoma: T-cell lymphomas were among the first clinical settings in which HDAC inhibitors were shown to be active. In a phase I trial of depsipeptide (romidepsin) conducted at the National Cancer Institute, 3 patients with cutane- 
ous T-cell lymphoma (CTCL) had a partial response, and 1 with peripheral T-cell lymphoma (PTCL) had a complete response. ${ }^{98}$ These observations provided the impetus for extensive clinical testing of romidepsin and other HDAC inhibitors in patients with Tcell lymphoma. In a phase II trial of oral vorinostat for refractory CTCL, 8 of 33 patients experienced partial response, including 7 with advanced disease and 4 with Sézary syndrome, while the time-to-disease progression was 30 weeks. ${ }^{99}$ The most common grade 3 or 4 drug-related adverse events were thrombocytopenia and dehydration. These results solidified the notion that vorinostat was active in heavily pretreated CTCL patients and identified the 400-mg daily regimen as the one with the most favorable safety profile. A multicenter phase IIb trial of vorinostat in persistent, progressive, or treatment-refractory CTCL, ${ }^{100}$ enrolled 64 patients who had undergone at least 2 prior systemic therapies (with at least 1 of which included bexarotene, unless intolerable) and showed an overall response rate of $29.7 \%$ and a median time-to-disease progression of 4.9 months. The results of these studies ${ }^{99,100}$ provided the basis for FDA approval of vorinostat for treating cutaneous manifestations of CTCL in patients with progressive, persistent, or recurrent disease while undergoing or after 2 systemic therapies. ${ }^{101}$ This effect of vorinostat on CTCL seems to be a class effect, as other HDAC inhibitors also seem to have clinical activity in that setting. For instance, panobinostat (LBH589) has also been shown to be active in this patient population. ${ }^{102}$

HDAC Inhibitors in Multiple Myeloma: The study of HDAC inhibitors in multiple myeloma (MM) was informed by preclinical data that showed that the HDAC inhibitor vorinostat (suberoylanilide hydroxamic acid) induces a constellation of antiproliferative and/or proapoptotic molecular events, including downregulation of transcripts for members of growth factor receptor signaling cascades, antiapoptotic molecules (e.g., caspase inhibitors), oncogenic kinases, DNA synthesis/repair enzymes, and transcription factors implicated in MM pathophysiology. ${ }^{103,104}$ These pleiotropic molecular events are associated with the ability of HDAC inhibitors to exhibit potent antiproliferative/proapoptotic activity against human MM cells, overcome the protective effect that bone marrow stromal cells have on MM cells, and enhance the response of MM cells to other anti-MM agents, including the proteasome inhibitor bortezomib. ${ }^{103,104}$
Based on these results and the favorable safety profile of oral administration of vorinostat in other disease settings, a phase I trial evaluated the safety and efficacy of oral vorinostat $(200,250$, or $300 \mathrm{mg}$ twice daily for 5 days per week in 4-week cycles; or 200, 300, or $400 \mathrm{mg}$ twice daily for 14 days in 3-week cycles) in patients with MM. In that trial, 13 patients (with a median of 3 lines of prior therapy) were evaluated. Treatment was continued until disease progression or intolerable toxicity was observed. ${ }^{105}$ Drug-related adverse events, which included fatigue, anorexia, dehydration, diarrhea, and nausea, were mostly grade 2 or below. Among 10 evaluable patients, 1 case of minimal response and 9 cases of stable disease were observed. Maximum tolerated doses were not determined due to early study termination by the sponsor. Although these observations might seem to indicate only modest single-agent activity of vorinostat in advanced MM, it is notable that the schedule of administration in this MM-specific phase I study was twicedaily. Clinical trials that led to vorinostat approval in CTCL showed that daily dosing is more active and better tolerated ${ }^{99}$ than twice-daily administration.

Given the preclinical observations regarding the anti-MM activity of the combination of vorinostat plus bortezomib, this combination was evaluated in clinical studies in advanced MM, including 2 separate multi-institutional phase I trials. ${ }^{106,107}$ Both studies confirmed clinical activity (with stable or decreasing M-protein in 13 of 16 and 17 of 17 patients, respectively) despite the heavily pretreated patient populations, which included most patients whose disease was refractory to the previous therapy; had received single or double autologous stem cell transplantation; and had previously received thalidomide-, lenalidomide-, or bortezomib-based therapies, including patients who had relapsed or whose disease was refractory to multiple lines of bortezomib-based treatment. Ongoing studies in the MM field are further evaluating the role of this combination in patients with bortezomib-refractory disease, whereas a randomized phase III trial is currently comparing vorinostat plus bortezomib versus bortezomib alone.

The preclinical and clinical observations regarding vorinostat activity in $\mathrm{MM}$ triggered interest for studies of other members of the HDAC inhibitor class in this setting. For instance, other hydroxamic acid HDAC inhibitors, such as LAQ824 ${ }^{108}$ and panobinostat (LBH589), ${ }^{109}$ were studied preclinically, 
Epigenetic Modulation in Hematologic Malignancies

while clinical studies of panobinostat in combination with bortezomib are also underway. A related line of research has involved the development of HDAC6-selective inhibitors, such as tubacin. ${ }^{110}$ This compound inhibits the tubulin deacetylation mediated by HDAC6. This cytoplasmic deacetylase helps transport misfolded proteins to the aggresome. Single-agent tubacin has limited in vitro anti-MM activity, but enhances the anti-MM activity of bortezomib. ${ }^{110}$ This effect is at least partly related to inhibition of aggresome function as a result of HDAC6 inhibition. Therefore, in contrast to hydroxamic acid inhibitors (vorinostat, LAQ824), tubacin primarily serves as a "cytosolic deacetylase" rather than a "nuclear deacetylase." 111 Therefore, despite some overlap between these 2 groups of functional activities (e.g., through LBH589-mediated inhibition of cytoplasmic deacetylation), ${ }^{109,112}$ it is conceivable that tubacin and other HDAC6-selective inhibitors primarily target cytoplasmic protein homeostasis, rather than functioning as classical examples of therapeutic targeting of epigenetic regulation at the level of chromatin remodeling.

HDAC Inhibitors in Other Hematologic Malignancies and MDS: HDAC inhibitors have been tested in clinical trials for patients with AML, ${ }^{113-115}$ chronic myeloid leukemia, ${ }^{113}$ acute lymphoblastic leukemia, ${ }^{113,115}$ chronic lymphocytic leukemia, ${ }^{113,116}$ MDS, ${ }^{113,117}$ and in lymphomas other than CTCL/ PTCL. ${ }^{118}$ These studies have shown that HDAC inhibitors do not generally exhibit substantial singleagent clinical activity (i.e., not to the level observed in CTCL/PTCL). Of note, several of these studies showed that HDAC inhibitor administration was associated with inhibition of the intended targets, as evidenced by hyperacetylation of histones, ${ }^{113}$ whereas in some cases differentiation or apoptosis of leukemic cells was also observed. ${ }^{116}$ The different pattern of responses to HDAC inhibition in CTCL/PTCL versus other hematologic malignancies, remains to be explained at the molecular level. It is conceivable that within each classically defined group of hematologic malignancies there are specific molecularly defined subtypes that are highly responsive to HDAC inhibition. Clinical trials with small numbers of patients may not be able to detect these specific molecularly defined subtypes. Modifications of dose/ schedule, as well as rational design of combination regimens, similar to the experience in the MM field, could conceivably extend the spectrum of activity of HDAC inhibitors. ${ }^{119,120}$

Nonchromatin-Related Sequelae of HDAC Inhibitors: HDAC inhibitors are a prime example of a therapeutic strategy that targets the aberrant epigenome of neoplastic cells. However, the molecular sequelae of HDAC inhibition extend well beyond the modulation of histone acetylation. In fact, there is now extensive evidence that agents such as vorinostat, panobinostat, and other HDAC inhibitors inhibit acetylation of a diverse range of nonhistone proteins implicated in proliferation, survival, and drug resistance of neoplastic cells (Figure 2). ${ }^{121}$

For instance, the function of HSP90, ${ }^{122} \mathrm{HIF}$ 1a, ${ }^{123}$ STAT3, ${ }^{124}$ p53, ${ }^{125,126}$ NF-kappaB subunits, ${ }^{127}$ and steroid hormone receptors ${ }^{128-130}$ is regulated by their acetylation status and is therefore influenced by the activity of HDACs. Consequently, HDAC inhibitors can mediate their antitumor effects through not only modulation of the histone code but also modifications in the activity of signaling cascades, the components of which are regulated by HDAC activity. Therefore, the term deacetylase inhibitors perhaps more accurately describes the properties of this drug class.

\section{Current Challenges and Future Directions}

A fundamental reason why targeting the epigenome represents an attractive anticancer strategy for diverse neoplasias is the pleiotropic range of molecular sequelae triggered by these therapies. Specifically, it is hoped that such strategies will be able to counteract the pronounced genetic complexity and heterogeneity of neoplastic cells. This appeal is further enhanced by the increasing number of "druggable" or potentially "druggable" therapeutic targets involved in the regulation of the epigenome in cancer cells. Although DNA methyltransferase inhibitors and HDAC inhibitors are already FDA approved for treatment of different types of neoplasias, mostly in the field of hematologic malignancies, other potentially druggable targets, including HAT or methyltransferase inhibitors, have also emerged in recent years.

However, a fundamental concern related to the pleiotropic nature of epigenetic therapies for cancer is that their molecular sequelae may not only occasionally include the derepression of tumor suppressor genes and/or inactivation of oncogenic transcripts, 


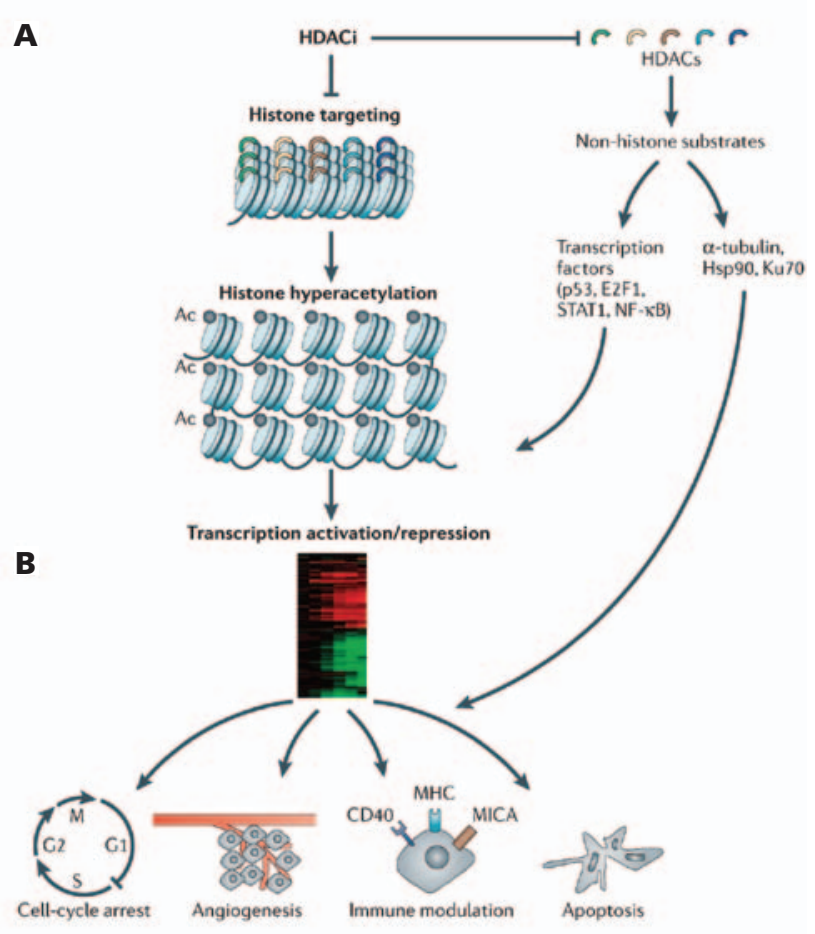

Figure 2 Effects of HDAC inhibition on nonhistone proteins. A) In addition to regulating the acetylation state of histones, HDAC can bind to, deacetylate, and regulate the activity of several other proteins, including transcription factors (i.e., $\mathrm{p} 53, \mathrm{E} 2 \mathrm{~F}$ transcription factor 1 [E2F1], and nuclear factor- $\mathrm{\kappa B}$ $[\mathrm{NF}-\kappa \mathrm{B}]$ ) and proteins with diverse biologic functions (i.e., $\alpha$-tubulin, Ku70, heat-shock protein 90 [hsp90]). Hyperacetylation of transcription factors with HDAC inhibitors (HDACis) can augment their gene-regulatory activities and contribute to the changes in gene expression observed after direct HDACi-mediated histone hyperacetylation. Hyperacetylation of proteins such as Ku70 and $\mathrm{Hsp} 90$, or disruption of protein phosphatase 1 (PP1)-HDAC interactions by HDACi might have no direct or indirect effect on gene expression but could be important for certain biologic effects of HDACi, in particular the induction of apoptosis and cell-cycle arrest. B) Antitumor effects of HDACi. HDACi can affect tumor cell growth and survival through multiple biologic effects. HDACi induce cell-cycle arrest at the G1/S boundary through upregulation of CDKN1A (encoding $\mathrm{p} 21^{\mathrm{WAF} / \mathrm{CIP1}}$ ) and/or through downregulation of cyclins. HDACi can suppress angiogenesis through decreased expression of proangiogenic factors, such as vascular endothelial growth factor (VEGF), hypoxia-inducible factor $1-\alpha(\mathrm{HIF} 1 \alpha)$, and chemokine (C-X-C motif) receptor 4 (CXCR4). HDACi can also have immunomodulatory effects by enhancing tumor cell antigenicity (upregulation of major histocompatibility complex class I and II, MICA, CD40) and by altering the expression of key cytokines, including tumor necrosis factor $\alpha$, interleukin-1, and interferon-gamma. Bolden JE, Peart MJ, Johnstone RW. Nat Rev Drug Disc 2006;5:769-784, copyright 2006. Reprinted by permission from Macmillan Publishers Ltd. but also may involve upregulation of some genes that could promote cell survival. ${ }^{3}$ One notable example of this concern involves the observation that ABC transporter genes (e.g., MDR1-P glycoprotein, ABCG2) are often transcriptionally activated in different types of tumor cells after exposure to HDAC inhibitors. ${ }^{131-136}$ This raises concerns about potential combinations of this drug class with conventional cytotoxic chemotherapy. Another example is the observation that, in biopsy specimens from 5 -azacitidine-treated patients with Epstein-Barr virus-associated tumors, significant demethylation was detected in all latent and lytic Epstein-Barr virus promoters examined within 72 hours of the conclusion of the last infusion of the first cycle of therapy, compared with pretreatment specimens. ${ }^{137}$ This experience, combined with anecdotal immunohistochemical evidence regarding activation of previously silent viral antigen expression, as well as case reports of EpsteinBarr virus or hepatitis $B$ virus reactivation in patients treated with romidepsin, ${ }^{138}$ suggests that more work may be necessary to exclude safety issues that may be caused by reactivation of latent viral infections.

Modifications of dose/schedule, as well as rational design of combination regimens are important strategies that can help expand the spectrum of activity of HDAC inhibitors and other epigenetic therapies. ${ }^{119,120}$ Combining these therapies with other existing drug classes requires careful study to avoid antagonistic effects that may be mediated by some of the many molecular sequelae triggered by pharmacologic modulators of epigenetic regulation of gene expression. This has been proposed as one of the reasons why it is important to develop epigenetic therapies with a higher degree of specificity towards individual target genes, as opposed to the currently available epigenetic therapies, the activity of which is considered to involve a more ubiquitous effect on the epigenome.

\section{Acknowledgments}

The authors apologize in advance for the inability, due to space limitations, to reference all studies pertinent to the topic of this manuscript. This activity is supported by an independent education grant from Merck. 
Epigenetic Modulation in Hematologic Malignancies

\section{References}

1. Balmain A, Gray J, Ponder B. The genetics and genomics of cancer. Nat Genet 2003;33:238-244.

2. Parsons DW, Jones S, Zhang X, et al. An integrated genomic analysis of human glioblastoma multiforme. Science 2008;321:18071812.

3. Piekarz RL, Bates SE. Epigenetic modifiers: basic understanding and clinical development. Clin Cancer Res 2009;15:3918-3926.

4. Mitsiades CS, McMillin DW, Klippel S, et al. The role of the bone marrow microenvironment in the pathophysiology of myeloma and its significance in the development of more effective therapies. Hematol Oncol Clin North Am 2007;21:1007-1034, vii-viii.

5. Jones PA, Baylin SB. The fundamental role of epigenetic events in cancer. Nat Rev Genet 2002;3:415-428.

6. Jenuwein T, Allis CD. Translating the histone code. Science 2001;293:1074-1080.

7. Luger K, Mader AW, Richmond RK, et al. Crystal structure of the nucleosome core particle at $2.8 \mathrm{~A}$ resolution. Nature 1997;389:251-260.

8. Gardiner-Garden M, Frommer M. CpG islands in vertebrate genomes. J Mol Biol 1987;196:261-282.

9. Constancia M, Pickard B, Kelsey G, Reik W. Imprinting mechanisms. Genome Res 1998;8:881-900.

10. Robertson KD, Jones PA. Dynamic interrelationships between DNA replication, methylation, and repair. Am J Hum Genet 1997;61:1220-1224.

11. Feinberg AP, Vogelstein B. Hypomethylation of ras oncogenes in primary human cancers. Biochem Biophys Res Commun 1983;111:47-54.

12. Feinberg AP, Vogelstein B. Hypomethylation distinguishes genes of some human cancers from their normal counterparts. Nature 1983;301:89-92.

13. Esteller M. CpG island hypermethylation and tumor suppressor genes: a booming present, a brighter future. Oncogene 2002;21:5427-5440.

14. Herman JG, Baylin SB. Gene silencing in cancer in association with promoter hypermethylation. N Engl J Med 2003;349:20422054.

15. Esteller M, Corn PG, Baylin SB, Herman JG. A gene hypermethylation profile of human cancer. Cancer Res 2001;61:3225-3229.

16. Eng C, Herman JG, Baylin SB. A bird's eye view of global methylation. Nat Genet 2000;24:101-102.

17. Egger G, Liang G, Aparicio A, Jones PA. Epigenetics in human disease and prospects for epigenetic therapy. Nature 2004;429:457-463.

18. Gaudet F, Hodgson JG, Eden A, et al. Induction of tumors in mice by genomic hypomethylation. Science 2003;300:489-492.

19. Eden A, Gaudet F, Waghmare A, Jaenisch R. Chromosomal instability and tumors promoted by DNA hypomethylation. Science 2003;300:455.

20. Howell CY, Bestor TH, Ding F, et al. Genomic imprinting disrupted by a maternal effect mutation in the Dnmt1 gene. Cell 2001;104:829-838.

21. Ulaner GA, Vu TH, Li T, et al. Loss of imprinting of IGF2 and H19 in osteosarcoma is accompanied by reciprocal methylation changes of a CTCF-binding site. Hum Mol Genet 2003;12:535549 .
22. Roman-Gomez J, Castillejo JA, Jimenez A, et al. 5' CpG island hypermethylation is associated with transcriptional silencing of the $\mathrm{p} 21$ (CIP1/WAF1/SDI1) gene and confers poor prognosis in acute lymphoblastic leukemia. Blood 2002;99:2291-2296.

23. Boldt DH. p21(CIP1/WAF1/SDI1) hypermethylation: an exciting new lead in ALL biology. Blood 2002;99:2283.

24. Sakai T, Toguchida J, Ohtani N, et al. Allele-specific hypermethylation of the retinoblastoma tumor-suppressor gene. Am J Hum Genet 1991;48:880-888.

25. Chim CS, Wong KY, Loong F, et al. Frequent epigenetic inactivation of Rb1 in addition to p15 and p16 in mantle cell and follicular lymphoma. Hum Pathol 2007;38:1849-1857.

26. Verdone L, Agricola E, Caserta M, Di Mauro E. Histone acetylation in gene regulation. Brief Funct Genomic Proteomic 2006;5:209-221.

27. Verdone L, Caserta M, Di Mauro E. Role of histone acetylation in the control of gene expression. Biochem Cell Biol 2005;83:344353.

28. Yang XJ. Lysine acetylation and the bromodomain: a new partnership for signaling. Bioessays 2004;26:1076-1087.

29. Fuks F, Burgers WA, Brehm A, et al. DNA methyltransferase Dnmt1 associates with histone deacetylase activity. Nat Genet 2000;24:88-91.

30. Bachman KE, Park BH, Rhee I, et al. Histone modifications and silencing prior to DNA methylation of a tumor suppressor gene. Cancer Cell 2003;3:89-95.

31. Espada J, Ballestar E, Fraga MF, et al. Human DNA methyltransferase 1 is required for maintenance of the histone $\mathrm{H} 3$ modification pattern. J Biol Chem 2004;279:37175-37184.

32. Lachner M, Jenuwein $\mathrm{T}$. The many faces of histone lysine methylation. Curr Opin Cell Biol 2002;14:286-298.

33. Lachner M, O'Sullivan RJ, Jenuwein T. An epigenetic road map for histone lysine methylation. J Cell Sci 2003;116:2117-2124.

34. Hake SB, Xiao A, Allis CD. Linking the epigenetic 'language' of covalent histone modifications to cancer. Br J Cancer 2004;90:761-769.

35. Esteller M. Epigenetics in cancer. N Engl J Med 2008;358:11481159.

36. Stirzaker C, Millar DS, Paul CL, et al. Extensive DNA methylation spanning the $\mathrm{Rb}$ promoter in retinoblastoma tumors. Cancer Res 1997;57:2229-2237.

37. Esteller M, Tortola S, Toyota M, et al. Hypermethylation-associated inactivation of p14(ARF) is independent of p16(INK4a) methylation and p53 mutational status. Cancer Res 2000;60:129133.

38. Foster SA, Wong DJ, Barrett MT, Galloway DA. Inactivation of p16 in human mammary epithelial cells by CpG island methylation. Mol Cell Biol 1998;18:1793-1801.

39. Esteller M, Fraga MF, Guo M, et al. DNA methylation patterns in hereditary human cancers mimic sporadic tumorigenesis. Hum Mol Genet 2001;10:3001-3007.

40. Kikuchi $\mathrm{T}$, Toyota M, Itoh $\mathrm{F}$, et al. Inactivation of $\mathrm{p} 57 \mathrm{KIP} 2$ by regional promoter hypermethylation and histone deacetylation in human tumors. Oncogene 2002;21:2741-2749.

41. Corn PG, Kuerbitz SJ, van Noesel MM, et al. Transcriptional silencing of the p73 gene in acute lymphoblastic leukemia and Burkitt's lymphoma is associated with 5' CpG island methylation. Cancer Res 1999;59:3352-3356. 
42. Kawano S, Miller CW, Gombart AF, et al. Loss of p73 gene expression in leukemias/lymphomas due to hypermethylation. Blood 1999;94:1113-1120.

43. Takita J, Yang HW, Chen YY, et al. Allelic imbalance on chromosome $2 q$ and alterations of the caspase 8 gene in neuroblastoma. Oncogene 2001;20:4424-4432.

44. Galm O, Wilop S, Reichelt J, et al. DNA methylation changes in multiple myeloma. Leukemia 2004;18:1687-1692.

45. Banelli B, Casciano I, Croce M, et al. Expression and methylation of CASP8 in neuroblastoma: identification of a promoter region. Nat Med 2002;8:1333-1335; author reply 1335.

46. Conway KE, McConnell BB, Bowring CE, et al. TMS1, a novel proapoptotic caspase recruitment domain protein, is a target of methylation-induced gene silencing in human breast cancers. Cancer Res 2000;60:6236-6242.

47. Stimson KM, Vertino PM. Methylation-mediated silencing of TMS1/ASC is accompanied by histone hypoacetylation and $\mathrm{CpG}$ island-localized changes in chromatin architecture. J Biol Chem 2002;277:4951-4958.

48. Galm O, Yoshikawa H, Esteller M, et al. SOCS-1, a negative regulator of cytokine signaling, is frequently silenced by methylation in multiple myeloma. Blood 2003;101:2784-2788.

49. Yoshikawa H, Matsubara K, Qian GS, et al. SOCS-1, a negative regulator of the JAK/STAT pathway, is silenced by methylation in human hepatocellular carcinoma and shows growth-suppression activity. Nat Genet 2001;28:29-35.

50. He B, You L, Uematsu K, et al. SOCS-3 is frequently silenced by hypermethylation and suppresses cell growth in human lung cancer. Proc Natl Acad Sci U S A 2003;100:14133-14138.

51. Niwa $Y$, Kanda H, Shikauchi $Y$, et al. Methylation silencing of SOCS-3 promotes cell growth and migration by enhancing JAK/ STAT and FAK signalings in human hepatocellular carcinoma. Oncogene 2005;24:6406-6417.

52. Esteller M, Guo M, Moreno V, et al. Hypermethylation-associated inactivation of the cellular retinol-binding-protein 1 gene in human cancer. Cancer Res 2002;62:5902-5905.

53. Taniguchi T, Tischkowitz M, Ameziane N, et al. Disruption of the Fanconi anemia-BRCA pathway in cisplatin-sensitive ovarian tumors. Nat Med 2003;9:568-574.

54. Melki JR, Vincent PC, Clark SJ. Concurrent DNA hypermethylation of multiple genes in acute myeloid leukemia. Cancer Res 1999;59:3730-3740.

55. Hegi ME, Liu L, Herman JG, et al. Correlation of O6-methylguanine methyltransferase (MGMT) promoter methylation with clinical outcomes in glioblastoma and clinical strategies to modulate MGMT activity. J Clin Oncol 2008;26:4189-4199.

56. Paz MF, Yaya-Tur R, Rojas-Marcos I, et al. CpG island hypermethylation of the DNA repair enzyme methyltransferase predicts response to temozolomide in primary gliomas. Clin Cancer Res 2004;10:4933-4938.

57. Herman JG, Latif F, Weng Y, et al. Silencing of the VHL tumorsuppressor gene by DNA methylation in renal carcinoma. Proc Natl Acad Sci U S A 1994;91:9700-9704.

58. Calvisi DF, Ladu S, Gorden A, et al. Mechanistic and prognostic significance of aberrant methylation in the molecular pathogenesis of human hepatocellular carcinoma. J Clin Invest 2007;117:2713-2722.

59. Kuzmin I, Geil L, Ge H, et al. Analysis of aberrant methylation of the VHL gene by transgenes, monochromosome transfer, and cell fusion. Oncogene 1999;18:5672-5679.
60. Bachman KE, Herman JG, Corn PG, et al. Methylation-associated silencing of the tissue inhibitor of metalloproteinase-3 gene suggest a suppressor role in kidney, brain, and other human cancers. Cancer Res 1999;59:798-802.

61. Kramer A, Schultheis B, Bergmann J, et al. Alterations of the cyclin D1/pRb/p16(INK4A) pathway in multiple myeloma. Leukemia 2002;16:1844-1851.

62. Cameron EE, Bachman KE, Myohanen S, et al. Synergy of demethylation and histone deacetylase inhibition in the re-expression of genes silenced in cancer. Nat Genet 1999;21:103-107.

63. Bruserud O, Stapnes C, Ersvaer E, et al. Histone deacetylase inhibitors in cancer treatment: a review of the clinical toxicity and the modulation of gene expression in cancer cell. Curr Pharm Biotechnol 2007;8:388-400.

64. Bruserud O, Stapnes C, Tronstad KJ, et al. Protein lysine acetylation in normal and leukaemic haematopoiesis: HDACs as possible therapeutic targets in adult AML. Expert Opin Ther Targets 2006;10:51-68.

65. Shilatifard A. Molecular implementation and physiological roles for histone H3 lysine 4 (H3K4) methylation. Curr Opin Cell Biol 2008;20:341-348.

66. Shilatifard A. Chromatin modifications by methylation and ubiquitination: implications in the regulation of gene expression. Annu Rev Biochem 2006; 75:243-269.

67. Esteller M, Gaidano G, Goodman SN, et al. Hypermethylation of the DNA repair gene $O(6)$-methylguanine DNA methyltransferase and survival of patients with diffuse large B-cell lymphoma. J Natl Cancer Inst 2002;94:26-32.

68. Esteller M, Garcia-Foncillas J, Andion E, et al. Inactivation of the DNA-repair gene MGMT and the clinical response of gliomas to alkylating agents. N Engl J Med 2000;343:1350-1354.

69. Hegi ME, Diserens AC, Gorlia T, et al. MGMT gene silencing and benefit from temozolomide in glioblastoma. N Engl J Med 2005;352:997-1003.

70. Gorlia T, van den Bent MJ, Hegi ME, et al. Nomograms for predicting survival of patients with newly diagnosed glioblastoma: prognostic factor analysis of EORTC and NCIC trial 26981 22981/CE.3. Lancet Oncol 2008;9:29-38.

71. Chim CS, Wong SY, Kwong YL. Aberrant gene promoter methylation in acute promyelocytic leukaemia: profile and prognostic significance. Br J Haematol 2003;122:571-578.

72. Shen L, Toyota M, Kondo Y, et al. Aberrant DNA methylation of p57KIP2 identifies a cell-cycle regulatory pathway with prognostic impact in adult acute lymphocytic leukemia. Blood 2003;101:4131-4136.

73. Uchida $T$, Kinoshita $T$, Ohno $T$, et al. Hypermethylation of p16INK4A gene promoter during the progression of plasma cell dyscrasia. Leukemia 2001;15:157-165.

74. Mateos MV, Garcia-Sanz R, Lopez-Perez R, et al. Methylation is an inactivating mechanism of the p16 gene in multiple myeloma associated with high plasma cell proliferation and short survival. Br J Haematol 2002;118:1034-1040.

75. $\mathrm{Ng} \mathrm{MH}$, To KW, Lo KW, et al. Frequent death-associated protein kinase promoter hypermethylation in multiple myeloma. Clin Cancer Res 2001;7:1724-1729.

76. Rosenfeld CS. Clinical development of decitabine as a prototype for an epigenetic drug program. Semin Oncol 2005;32:465-472.

77. Fenaux P, Mufti GJ, Hellstrom-Lindberg E, et al. Efficacy of azacitidine compared with that of conventional care regimens in the 
Epigenetic Modulation in Hematologic Malignancies

treatment of higher-risk myelodysplastic syndromes: a randomized, open-label- phase III study. Lancet Oncol 2009;10:223-232.

78. Kantarjian HM, O'Brien S, Huang X, et al. Survival advantage with decitabine versus intensive chemotherapy in patients with higher risk myelodysplastic syndrome: comparison with historical experience. Cancer 2007;109:1133-1137.

79. Issa JP, Garcia-Manero G, Giles FJ, et al. Phase 1 study of lowdose prolonged exposure schedules of the hypomethylating agent 5-aza-2'-deoxycytidine (decitabine) in hematopoietic malignancies. Blood 2004;103:1635-1640.

80. Muller-Thomas C, Schuster T, Peschel C, Gotze KS. A limited number of 5-azacitidine cycles can be effective treatment in MDS. Ann Hematol 2009;88:213-219.

81. Maslak P, Chanel S, Camacho LH, et al. Pilot study of combination transcriptional modulation therapy with sodium phenylbutyrate and 5-azacytidine in patients with acute myeloid leukemia or myelodysplastic syndrome. Leukemia 2006;20:212-217.

82. Nand S, Godwin J, Smith S, et al. Hydroxyurea, azacitidine and gemtuzumab ozogamicin therapy in patients with previously untreated non-M3 acute myeloid leukemia and high-risk myelodysplastic syndromes in the elderly: results from a pilot trial. Leuk Lymphoma 2008;49:2141-2147.

83. Griffiths EA, Gore SD. DNA methyltransferase and histone deacetylase inhibitors in the treatment of myelodysplastic syndromes. Semin Hematol 2008;45:23-30.

84. De Padua Silva L, de Lima M, Kantarjian H, et al. Feasibility of allo-SCT after hypomethylating therapy with decitabine for myelodysplastic syndrome. Bone Marrow Transplant 2009;43:839-843.

85. Oki Y, Kantarjian HM, Gharibyan V, et al. Phase II study of lowdose decitabine in combination with imatinib mesylate in patients with accelerated or myeloid blastic phase of chronic myelogenous leukemia. Cancer 2007;109:899-906.

86. Blum W, Klisovic RB, Hackanson B, et al. Phase I study of decitabine alone or in combination with valproic acid in acute myeloid leukemia. J Clin Oncol 2007;25:3884-3891.

87. Soriano AO, Yang H, Faderl S, et al. Safety and clinical activity of the combination of 5-azacytidine, valproic acid, and all-trans retinoic acid in acute myeloid leukemia and myelodysplastic syndrome. Blood 2007;110:2302-2308.

88. Quintas-Cardama A, Tong W, Kantarjian H, et al. A phase Il study of 5-azacitidine for patients with primary and post-essential thrombocythemia/polycythemia vera myelofibrosis. Leukemia 2008;22:965-970.

89. Wijermans PW, Ruter B, Baer MR, et al. Efficacy of decitabine in the treatment of patients with chronic myelomonocytic leukemia (CMML). Leuk Res 2008;32:587-591.

90. Garcia-Manero G. Demethylating agents in myeloid malignancies. Curr Opin Oncol 2008;20:705-710.

91. Lozzio CB, Lozzio BB, Machado EA, et al. Effects of sodium butyrate on human chronic myelogenous leukaemia cell line K562. Nature 1979;281:709-710.

92. Hoffman R, Murnane MJ, Benz EJ Jr, et al. Induction of erythropoietic colonies in a human chronic myelogenous leukemia cell line. Blood 1979;54:1182-1187.

93. Baker PN, Morser J, Burke DC. Effects of sodium butyrate on a human lymphoblastoid cell line (Namalwa) and its interferon production. J Interferon Res 1980;1:71-77.

94. Bode J, Hochkeppel HK, Maass K. Links between effects of butyrate on histone hyperacetylation and regulation of inter- feron synthesis in Namalva and FS-4 cell lines. J Interferon Res 1982;2:159-166.

95. Kruh J. Effects of sodium butyrate, a new pharmacological agent, on cells in culture. Mol Cell Biochem 1982;42:65-82.

96. Gore SD, Carducci MA. Modifying histones to tame cancer: clinical development of sodium phenylbutyrate and other histone deacetylase inhibitors. Expert Opin Investig Drugs 2000;9:2923 2934.

97. Prince HM, Bishton MJ, Harrison SJ. Clinical studies of histone deacetylase inhibitors. Clin Cancer Res 2009;15:3958-3969.

98. Piekarz RL, Robey R, Sandor V, et al. Inhibitor of histone deacetylation, depsipeptide (FR901228), in the treatment of peripheral and cutaneous T-cell lymphoma: a case report. Blood 2001;98:2865-2868.

99. Duvic M, Talpur R, Ni X, et al. Phase 2 trial of oral vorinostat (suberoylanilide hydroxamic acid, SAHA) for refractory cutaneous T-cell lymphoma (CTCL). Blood 2007;109:31-39.

100. Olsen EA, Kim YH, Kuzel TM, et al. Phase IIb multicenter trial of vorinostat in patients with persistent, progressive, or treatment refractory cutaneous T-cell lymphoma. J Clin Oncol 2007;25:3109_ 3115.

101. Mann BS, Johnson JR, Cohen MH, et al. FDA approval summary: vorinostat for treatment of advanced primary cutaneous T-cell lymphoma. Oncologist 2007;12:1247-1252.

102. Ellis L, Pan Y, Smyth GK, et al. Histone deacetylase inhibitor panobinostat induces clinical responses with associated alterations in gene expression profiles in cutaneous T-cell lymphoma. Clin Cancer Res 2008;14:4500-4510.

103. Mitsiades CS, Mitsiades NS, McMullan CJ, et al. Transcriptional signature of histone deacetylase inhibition in multiple myeloma: biological and clinical implications. Proc Natl Acad Sci U S A 2004;101:540-545.

104. Mitsiades N, Mitsiades CS, Richardson PG, et al. Molecular sequelae of histone deacetylase inhibition in human malignant $\mathrm{B}$ cells. Blood 2003;101:4055-4062.

105. Richardson P, Mitsiades C, Colson K, et al. Phase I trial of oral vorinostat (suberoylanilide hydroxamic acid, SAHA) in patients with advanced multiple myeloma. Leuk Lymphoma 2008;49:502_ 507.

106. Badros A, Philip S, Niesvizky R, et al. Phase I trial of suberoylanilide hydroxamic acid (SAHA) plus bortezomib (Bort) in relapsed multiple myeloma (MM) patients (pts). Blood 2007;110:354A354A.

107. Weber DM, Jagannath S, Mazumder A, et al. Phase I trial of oral vorinostat (Suberoylanilide hydroxamic acid, SAHA) in combination with bortezomib in patients with advanced multiple myeloma. Blood 2007;110:355A-355A.

108. Catley L, Weisberg E, Tai YT, et al. NVP-LAQ824 is a potent novel histone deacetylase inhibitor with significant activity against multiple myeloma. Blood 2003;102:2615-2622.

109. Catley L, Weisberg E, Kiziltepe T, et al. Aggresome induction by proteasome inhibitor bortezomib and alpha-tubulin hyperacetylation by tubulin deacetylase (TDAC) inhibitor LBH589 are synergistic in myeloma cells. Blood 2006;108:3441-3449.

110. Hideshima T, Bradner JE, Wong J, et al. Small-molecule inhibition of proteasome and aggresome function induces synergistic antitumor activity in multiple myeloma. Proc Natl Acad Sci U S A 2005;102:8567-8572.

111. Haggarty SJ, Koeller KM, Wong JC, et al. Domain-selective smallmolecule inhibitor of histone deacetylase 6 (HDAC6)-mediated 
tubulin deacetylation. Proc Natl Acad Sci U S A 2003;100:4389_ 4394.

112. Bali P, Pranpat M, Bradner J, et al. Inhibition of histone deacetylase 6 acetylates and disrupts the chaperone function of heat shock protein 90: a novel basis for antileukemia activity of histone deacetylase inhibitors. J Biol Chem 2005;280:26729-26734.

113. Garcia-Manero G, Yang H, Bueso-Ramos C, et al. Phase 1 study of the histone deacetylase inhibitor vorinostat (suberoylanilide hydroxamic acid [SAHA]) in patients with advanced leukemias and myelodysplastic syndromes. Blood 2008;111:1060-1066.

114. Odenike OM, Alkan S, Sher D, et al. Histone deacetylase inhibitor romidepsin has differential activity in core binding factor acute myeloid leukemia. Clin Cancer Res 2008;14:7095-7101.

115. Giles F, Fischer T, Cortes J, et al. A phase I study of intravenous LBH589, a novel cinnamic hydroxamic acid analogue histone deacetylase inhibitor, in patients with refractory hematologic malignancies. Clin Cancer Res 2006;12:4628-4635.

116. Byrd JC, Marcucci G, Parthun MR, et al. A phase 1 and pharmacodynamic study of depsipeptide (FK228) in chronic lymphocytic leukemia and acute myeloid leukemia. Blood 2005;105:959-967.

117. Klimek VM, Fircanis S, Maslak P, et al. Tolerability, pharmacodynamics, and pharmacokinetics studies of depsipeptide (romidep$\sin$ ) in patients with acute myelogenous leukemia or advanced myelodysplastic syndromes. Clin Cancer Res 2008;14:826-832.

118. Kelly WK, Richon VM, O'Connor O, et al. Phase I clinical trial of histone deacetylase inhibitor: suberoylanilide hydroxamic acid administered intravenously. Clin Cancer Res 2003;9:3578-3588.

119. Ellis L, Atadja PW, Johnstone RW. Epigenetics in cancer: targeting chromatin modifications. Mol Cancer Ther 2009;8:1409_ 1420.

120. Bots M, Johnstone RW. Rational combinations using HDAC inhibitors. Clin Cancer Res 2009;15:3970-3977.

121. Bolden JE, Peart MJ, Johnstone RW. Anticancer activities of histone deacetylase inhibitors. Nat Rev Drug Disc 2006;5:769-784.

122. Aoyagi S, Archer TK. Modulating molecular chaperone Hsp90 functions through reversible acetylation. Trends Cell Biol 2005;15:565-567.

123. Jeong JW, Bae MK, Ahn MY, et al. Regulation and destabilization of HIF-1alpha by ARD1-mediated acetylation. Cell 2002;111:709-720.

124. O'Shea JJ, Kanno Y, Chen X, Levy DE. Cell signaling. Stat acetylation - a key facet of cytokine signaling? Science 2005;307:217218.

125. Luo J, Su F, Chen D, et al. Deacetylation of p53 modulates its effect on cell growth and apoptosis. Nature 2000;408:377-381.
126. Insinga A, Monestiroli S, Ronzoni S, et al. Impairment of p53 acetylation, stability and function by an oncogenic transcription factor. Embo J 2004;23:1144-1154.

127. Quivy V, Van Lint C. Regulation at multiple levels of NF-kappaBmediated transactivation by protein acetylation. Biochem Pharmacol 2004;68:1221-1229.

128. Fu M, Rao M, Wang C, et al. Acetylation of androgen receptor enhances coactivator binding and promotes prostate cancer cell growth. Mol Cell Biol 2003;23:8563-8575.

129. Mazumdar A, Wang RA, Mishra SK, et al. Transcriptional repression of oestrogen receptor by metastasis-associated protein 1 corepressor. Nat Cell Biol 2001;3:30-37.

130. Wang C, Fu M, Angeletti RH, et al. Direct acetylation of the estrogen receptor alpha hinge region by $\mathrm{p} 300$ regulates transactivation and hormone sensitivity. J Biol Chem 2001;276:1837518383.

131. Robey RW, Zhan Z, Piekarz RL, et al. Increased MDR1 expression in normal and malignant peripheral blood mononuclear cells obtained from patients receiving depsipeptide (FR901228, FK228, NSC630176). Clin Cancer Res 2006;12:1547-1555.

132. Jin S, Scotto KW. Transcriptional regulation of the MDR1 gene by histone acetyltransferase and deacetylase is mediated by NF-Y. Mol Cell Biol 1998;18:4377-4384.

133. Tabe Y, Konopleva M, Contractor R, et al. Up-regulation of MDR1 and induction of doxorubicin resistance by histone deacetylase inhibitor depsipeptide (FK228) and ATRA in acute promyelocytic leukemia cells. Blood 2006;107:1546-1554.

134. To KK, Polgar O, Huff LM, et al. Histone modifications at the ABCG2 promoter following treatment with histone deacetylase inhibitor mirror those in multidrug-resistant cells. Mol Cancer Res 2008;6:151-164.

135. Yatouji S, El-Khoury V, Trentesaux C, et al. Differential modulation of nuclear texture, histone acetylation, and MDR1 gene expression in human drug-sensitive and -resistant OV1 cell lines. Int J Oncol 2007;30:1003-1009.

136. Hauswald S, Duque-Afonso J, Wagner MM, et al. Histone deacetylase inhibitors induce a very broad, pleiotropic anticancer drug resistance phenotype in acute myeloid leukemia cells by modulation of multiple ABC transporter genes. Clin Cancer Res 2009; 15:3705-3715.

137. Chan AT, Tao Q, Robertson KD, et al. Azacitidine induces demethylation of the Epstein-Barr virus genome in tumors. J Clin Oncol 2004;22:1373-1381.

138. Ritchie D, Piekarz RL, Blombery P, et al. Reactivation of DNA viruses in association with histone deacetylase inhibitor (HDI) therapy: a case series report. Haematologica 2009; in press. 